\title{
Effect of Abdominal Massage on Feeding Intolerance of Premature Infants
}

\author{
Xin Jin, Lilan He ${ }^{*}$, Shiyi Zhang, Chuanrui Zhu \\ Nursing Department, The First Affiliated Hospital, Jinan University, Guangzhou, China \\ Email address: \\ 724514781@qq.com (Xin Jin), helilan@yeah.net (Lilan He) \\ ${ }^{*}$ Corresponding author
}

To cite this article:

Xin Jin, Lilan He, Shiyi Zhang, Chuanrui Zhu. Effect of Abdominal Massage on Feeding Intolerance of Premature Infants. American Journal of Nursing Science. Vol. 9, No. 4, 2020, pp. 196-199. doi: 10.11648/j.ajns.20200904.14

Received: May 3, 2020; Accepted: May 25, 2020; Published: June 3, 2020

\begin{abstract}
Aim: To evaluate the improvement of feeding intolerance to premature infants by abdominal massage. Method: From January 2018 to January 2020, the basic data of 72 premature infants in the NICU of our hospital were collected by retrospective method, which was divided into an experimental group and control group, 36 cases ineach group. The infants in the experimental group received such intervention of abdominal massage with daily fixed time and fixed frequency, and the control group without abdominal massage. The gestational age, birth weight, sex was not statistically significant in the two groups. The outcome indexes of the two groups were evaluated including the length of stay, the weight beginning oral feeding, the weight reaching total oral feeding, the weight at discharge, transition time, time to recover to birth weight, the daily weight gain, gastric residue. Results: Data analysis by SPSS24.0 showed that the weight beginning oral feeding $(P=0.012)$, weight reaching total oral feeding $(\mathrm{P}=0.014)$ and the mean times of gastric residue in the experimental group was statistically significant. But there was no significant difference in the length of stay, the weight at discharge, transition time, time to recover to birth weight, and the daily weight gain. Conclusion: Intervention of abdominal massage might promote the weight gain and improve the feeding intolerance in premature infants.
\end{abstract}

Keywords: Premature Infants, Abdominal Massage, Feeding Intolerance

\section{Introduction}

There are approximately 15 million premature infants were born before 37 weeks every year around the world [1]. However, due to various immature systems, most premature infants are prone to gastrointestinal dysfunction, and have the higher incidence of feeding intolerance. Feeding intolerance refers to the occurrence of vomiting, abdominal distension, and high gastric residual volume [2], which affected the establishment of enteral nutrition and prolonged use of parenteral nutrition in premature infants. On this account, they further obtained impaired nutritional supply and achieved adverse growth and neurodevelopment due to the increased risk of infection, the occurrence of narcotizing enterocolitis (NEC), perforation of the intestine and the cholestasis [3-5]. Because of the necessary special colony of premature infants, especially with low birth quality, reasonable nutrition supply, improving the gastrointestinal function and optimizing their prognosis are significant challenges [6].

Abdominal massage is a useful way to relax the abdominal muscles of infants to the greatest extent, which could effectively relieve their gastrointestinal discomfort. Studies $[7,8]$ have shown that massage could not only promote the early growth and development of premature infants, but also greatly increase the levels of gastrin and insulin in premature infants, thus increasing the digestion and absorption of their nutrients. Meanwhile, during abdominal massage therapeutic signals could be transmitted directly to brain by means of skin contact and pressure, stimulating the vagus nerve, thus promoting intestinal peristalsis [9-11]. However, little research showed the connection between intervention of abdominal massage and feeding intolerance in premature infants. The aim of our study was to evaluate the improvement of feeding intolerance to premature infants in the Neonatal Intensive Care Unit (NICU) through the abdominal massage. 


\section{Method}

\subsection{Study Design}

We used a retrospective control method to select the premature infants born in the first affiliated hospital of Jinan University and then transferred to the NICU from January 2018 to January 2020 . The gestational age was ranged from 28 to 32 weeks and the birth weight was ranged from 1 to 2.5 kilograms. A total of 36 premature infants had received abdominal massage during this period after excluding the people with major congenital anomalies, gastrointestinal anomalies and hypoxic-ischemic encephalopathy. 36 premature infants were matched to control group without abdominal massage during that period under the same exclusion criteria.

\subsection{Details of Abdominal Massage}

The intervenor is three registered doctors or registered nurses in NICU. They all have received unified training before operation, including massage manipulation, massage time, massage location and massage intensity. The room temperature of environmental requirements for massage is $26-28^{\circ} \mathrm{C}$, and the humidity was $55 \%-65 \%$. All premature infants in the experimental group got abdominal massage started 24 hours after birth, and the massage time and frequency were determined according to literature research and expert opinion. Twice a day and lasting 15 minutes when enema operation time, 9:00 AM and 5:00 PM respectively, which is also between half an hour to an hour before the meal for premature infants, avoiding vomiting. Both the experimental group and the control group had glycerin and saline solution. The ratio of glycerol to salt water was $1: 1.5$.

Premature infants were placed on the baby's open table during the intervention. We maintained the table temperature between 36.5 and $37.5^{\circ} \mathrm{C}$ adjusted to the skin temperature. Took the babies in the head high foot low supine position, and both legs were in natural frog shape. Opened the diapers with attention to the privacy of them. Used $5 \mathrm{Ml}$ syringes to suction solution, and dipped a little paraffin oil into syringe suppository. Then syringe hold by the left hand directly inserted into the anus and injected slowly, and after stayed for 1 minute pulled out the syringe with pinching the infants' hips to avoid a few seconds by the right hand. Finally, took off the gloves. Control group operation ended here, and intervention group started operation last about 15 minutes. We put a drop of a little Johnson baby moisturizing oil (U.S. 100 years baby care brands) in the hands, with hands friction to warm the hands. Then gently pressed the abdomen avoiding the umbilical cord through finger abdomen, and from the right lower of abdomen upward slowly with hands alternately, to the rib transverse, to the left rib then downward, according to the infants' intestinal direction touch. It appeared as the shape of inverted "I LOVE YOU".

\subsection{Outcome Indicators}

The outcome indicators including: length of stay, the weight beginning oral feeding, the weight reaching total oral feeding (Oral feeding up to $120 \mathrm{ml} / \mathrm{kg} / \mathrm{d}$, without nasal feeding up to 48 hours)[12], the weight at discharge, transition time (the time from beginning oral feeding to total oral feeding), time to recover to birth weight, the daily weight gain [13] (the daily weight gain $[\mathrm{g} /(\mathrm{kg} \times \mathrm{d})]=[1000 \times \mathrm{In}$ (weight at discharge $/$ birth weight) $] /($ length of stay - time to recover to birth weight), gastric residue. Since it was a retrospective control study, the duration of enema in each premature infant was different. The longest duration of enema in all data was 59 days, with the minimum enema days was 7 days, in order to reduce the influence of interference factors, the gastric residue was recorded from the first day to the seventh day both in two groups.

\subsection{Data Analysis}

We used the Statistical Package for Social Sciences (SPSS), version 24.0 to analyse the data. All the data were carried out using The homogeneity test (X2), Independent t-test or The Wilcoxon signed test. Set the confidence interval to $95 \%$, and $\mathrm{p}<0.05$ was considered to be statistically.

\section{Result}

Table 1. Characteristics of preterm infants.

\begin{tabular}{llll}
\hline & Experimental group & Control group & Statistical significance \\
\hline Gestational age (week) & $29.69 \pm 1.56$ & $30.03 \pm 1.48$ & $\mathrm{t}=-0.928$ \\
& & & $\mathrm{P}=0.357$ \\
Birth weight (Kg) & $1.45 \pm 0.31$ & $1.38 \pm 0.22$ & $\mathrm{t}=1.186$ \\
& & & $\mathrm{P}=0.240$ \\
Gender & & & \\
Male n (\%) & $22(61.1)$ & $25(69.4)$ & $\mathrm{X} 2=0.551$ \\
Female $\mathrm{n}(\%)$ & $14(38.9)$ & $11(30.6)$ & $\mathrm{P}=0.458$ \\
\hline
\end{tabular}

The characteristics of the experimental group and the control group are summarized in Table 1. It showed that no significant differences were observed comparing two groups in terms of gestational age, birth weight and gender. 
Table 2. Comparision of indicators for two groups.

\begin{tabular}{|c|c|c|c|}
\hline & Experimental group & Control group & Statistical significance \\
\hline \multirow[t]{2}{*}{ Length of stay (day) } & $47.17 \pm 16.15$ & $49.03 \pm 16.23$ & $\mathrm{t}=-0.485$ \\
\hline & & & $P=0.629$ \\
\hline \multirow[t]{2}{*}{ Transition time (day) } & $5.81[1.25,8.75]$ & $5.25[1.25,8.75]$ & $z=-0.645$ \\
\hline & & & $\mathrm{P}=0.519$ \\
\hline \multirow[t]{2}{*}{ The weight beginning oral feeding $(\mathrm{Kg})$} & $1.89 \pm 1.93$ & $1.78 \pm 1.83$ & $\mathrm{t}=2.568$ \\
\hline & & & $\mathrm{p}=0.012$ \\
\hline \multirow[t]{2}{*}{ The weight reaching total oral feeding $(\mathrm{Kg})$} & 2. $03 \pm 0.22$ & $1.89 \pm 0.24$ & $\mathrm{t}=2.525$ \\
\hline & & & $\mathrm{p}=0.014$ \\
\hline \multirow[t]{2}{*}{ The weight at discharge $(\mathrm{Kg})$} & 2. $26 \pm 0.24$ & $2.22 \pm 0.19$ & $\mathrm{t}=0.679$ \\
\hline & & & $\mathrm{p}=0.500$ \\
\hline \multirow[t]{2}{*}{ time to recover to birth weight (day) } & $9.03 \pm 4.802$ & $9.06 \pm 5.104$ & $\mathrm{t}=-0.024$ \\
\hline & & & $\mathrm{p}=0.981$ \\
\hline \multirow[t]{2}{*}{ the daily weight gain $(\mathrm{g} / \mathrm{Kg} * \mathrm{~d})$} & 13. $38[10.44,13.67]$ & 12. $76[10.33,16.79]$ & $z=-1.070$ \\
\hline & & & $\mathrm{p}=0.285$ \\
\hline
\end{tabular}

Table 3. Comparison of the mean times of gastric residue per infant during the first seven days after abdominal massage.

\begin{tabular}{lllll}
\hline & No gastric remnant & The volume $\leq \mathbf{1 0} \%$ & The volume $>\mathbf{1 0} \%$ and $\leq \mathbf{5 0 \%}$ & The volume $\geq \mathbf{5 0 \%}$ \\
\hline Experimental group & $41.00 \pm 14.51$ & $0.13 \pm 0.17$ & $2.42 \pm 3.73$ & $5.44 \pm 5.63$ \\
Control group & $37.47 \pm 10.97$ & $0.47 \pm 0.97$ & $6.33 \pm 6.12$ & $9.75 \pm 9.14$ \\
& $\mathrm{t}=1.164$ & $\mathrm{t}=-2.708$ & $\mathrm{t}=-3.278$ & $\mathrm{t}=-2.406$ \\
& $\mathrm{p}=0.249$ & $\mathrm{p}=0.009$ & $\mathrm{p}=0.002$ & $\mathrm{p}=0.019$ \\
\hline
\end{tabular}

There were no significant differences in two groups in terms of length of stay, transition time, the weight at discharge, time to recover to birth weight, the daily weight gain $(\mathrm{p}>0.05)$. But the weight beginning oral feeding and the weight reaching total oral feeding was significantly differences between two groups $(\mathrm{p}<0.05)$. Details showed in table 2 .

In table 3, we observed that the mean times of the gastric remnant in the experimental group is significantly lower than that in the control group, and the difference was statistically significant during the first seven days $(p<0.05)$. And the experimental group has higher times of no gastric remnant, though no significant difference compared with control group.

\section{Discussion}

According to the growth and development characteristics of the fetus, gastrointestinal differentiation stated from the 28th gestational week, the small intestine began to have functional peristalsis slowly at the 30th gestational week, and the production of systemic intestinal peristalsis at the 34th gestational week [14]. Therefore, the function of suck, swallow, absorb and digest in low birth weight infants is poor, with smaller stomach, slower gastrointestinal peristalsis, which is prone to feeding intolerance phenomenon such as choking, vomiting, abdominal distension, weight loss for a long time and other. In our study, the average number of gastric residues in the massage group was significantly lower than that in the control group, which was consistent with the results of the meta analysis [15] of Leil. Parasympathetic stimulation is one of the neural mechanisms that may help improve feeding intolerance. Studies [9] have shown that massage can stimulate gastrointestinal system and parasympathetic activity, increase the secretion of gastrointestinal peptide hormones, make gastrointestinal structure function more mature, reduce the occurrence of intolerance, and absorb nutrients more fully. In this study, although there was no significant difference in the daily weight gain between the two groups, the weight beginning oral feeding and reaching total oral feeding in the massage group was significantly higher than that in the control group. The results showed that massage might improve the weight gain of low birth weight infants.

In terms of the indicator of recovery to birth weight, the recovery stage after the lowest body mass reflects the nutritional absorption of premature infants. Xie et al.[14] used abdominal massage to the premature infants at 29th to 33th gestational weeks, and it was found that although the average hospitalization time of infants who received abdominal massage was not significantly shorter than that of those who did not receive it, the time to return to birth weight was significantly shorter. In our study, although there was not any significant statistical difference in those indicators between the two groups, the mean time in the experimental group was still shorter than that in the control group. In addition, there was no significant improvement in the transition time by massage in this study, which may be related not only to the digestion and absorption ability of the infants, but also to the oral exercise ability of them. A more coordinated swallowing-sucking pattern is more conducive to the transition from oral feeding to full oral feeding.

By means of the textbook and literature search, we trained the intervenors to get the coincident massage manipulation, 
massage time, massage location and massage intensity. The intervenors make the infants feel safe and comfortable. The results showed that the abdominal massage can not only improve the feeding intolerance of infants, but also promote the intimate relationship between the operator and the infants, so that they were filled with a sense of safety and were beneficial to the their family return and healthy growth.

\section{Conclusion}

Intervention of abdominal massage might promote the weight gain and improve the feeding intolerance in premature infants. As a low-cost and cost-effective intervention, abdominal massage could effectively improve the gastrointestinal intolerance of infants and accelerate their growth, which could be considered for the clinic. Since it is a retrospective study, data are not completed such as frequency of vomiting, time of meconium emptying. Higher quality experimental methods, longer interventions and larger sample sizes could be designed in the future to ensure the effect of massage on feeding intolerance in these low birth weight infants.

\section{References}

[1] Kamphorst K, Sietsma Y, Brouwer AJ, et al. Enemas, suppositories and rectal stimulation are not effective in accelerating enteral feeding or meconium evacuation in low-birthweight infants: a systematic review. Acta Paediatr, 2016, 105 (11): 1280-1287.

[2] Tekgunduz KS, Gurol A, Apay SE, et al. Effect of abdomen massage for prevention of feeding intolerance in preterm infants. Ital J Pediatr, 2014, 40: 89-89.

[3] Mohammadizadeh M, Ghazinour M, Iranpour R. Efficacy of prophylactic oral erythromycin to improve enteral feeding tolerance in preterm infants: a randomised controlled study. Singapore Med J, 2010, 51 (12): 952-956.

[4] Mena NP, Leon Del PJ, Sandino PD, et al. [Meconium evacuation to improve feeding tolerance in very low birth weight preterm infants (Emita Protocol)]. Rev Chil Pediatr, 2014, 85 (3): 304-311.

[5] Ibrahim T, Li Wei C, Bautista D, et al. Saline Enemas versus Glycerin Suppositories to Promote Enteral Feeding in Premature Infants: A Pilot Randomized Controlled Trial. Neonatology, 2017, 112 (4): 347-353.

[6] Lucchini R, Bizzarri B, Giampietro S, et al. Feeding intolerance in preterm infants. How to understand the warning signs. J Matern Fetal Neonatal Med, 2011, 24 Suppl 1: 72-74.

[7] Chen LL, Su YC, Su CH, et al. Acupressure and meridian massage: combined effects on increasing body weight in premature infants. J Clin Nurs, 2008, 17 (9): 1174-1181.

[8] Massaro AN, Hammad TA, Jazzo B, et al. Massage with kinesthetic stimulation improves weight gain in preterm infants. J Perinatol, 2009, 29 (5): 352-357.

[9] Diego MA, Field T, Hernandez-Reif M. Vagal activity, gastric motility, and weight gain in massaged preterm neonates. $\mathrm{J}$ Pediatr, 2005, 147 (1): 50-55.

[10] Carter BM, Howard C. A 6th Vital Sign--Potential Use of Nasogastric Tube for Intra-abdominal Pressure Monitoring Method to Detect Feeding Intolerance in Very Low Birth-Weight Preterm Infants $(<1500$ g). Adv Neonatal Care, 2015, 15 (3): 176-181.

[11] Diego MA, Field T, Hernandez-Reif M, et al. Preterm infant massage elicits consistent increases in vagal activity and gastric motility that are associated with greater weight gain. Acta Paediatr, 2007, 96 (11): 1588-1591.

[12] Li FN, Li XR, Xue WS, et al. Effect of Maternal Sound Stimulation on oral feeding among premature infants. Chinese Nursing Management, 2016, (04): 481-485.

[13] Patel AL, Engstrom JL, Meier PP, et al. Accuracy of methods for calculating postnatal growth velocity for extremely low birth weight infants. Pediatrics, 2005, 116 (6): 1466-1473.

[14] Xie YQ, Liu WQ. Effect of cluster nursing combined with abdominal massage on feeding premature infants with low birth weight. Fujian Med J, 2018, 40 (06): 154-155.

[15] Seiiedi-Biarag L, Mirghafourvand M. The effect of massage on feeding intolerance in preterm infants: a systematic review and meta-analysis study. Ital J Pediatr, 2020, 46 (1): 52-52. 